\title{
Are cranial ultrasound requests and reports adequate at two academic hospitals in Johannesburg?
}

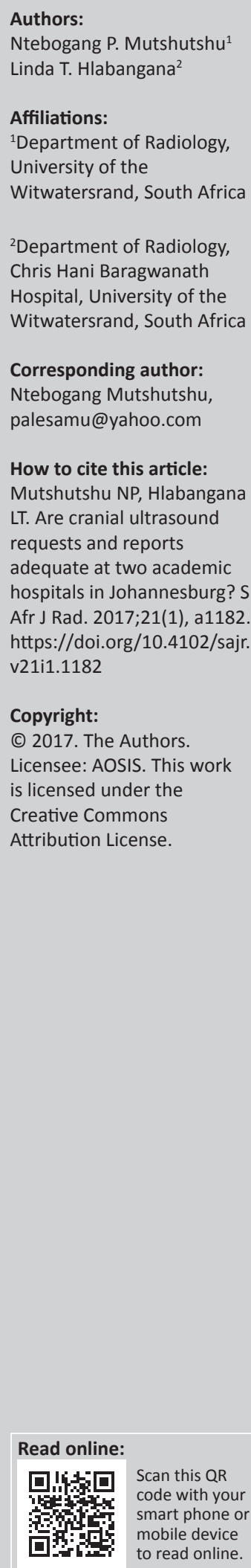

Background: Cranial ultrasound is a cheap, effective and easy to use modality for the evaluation of cranial pathology in very sick paediatric populations. It can be performed as a portable imaging investigation and repeated as many times as possible. New improvements in sonography equipment and technique make it possible for cranial ultrasound to compete with CT scanners in terms of identifying pathology.

Aim: To determine the adequacy of cranial ultrasound requests and reports at Charlotte Maxeke Johannesburg Academic (CMJAH) and Rahima Moosa Mother and Child Hospitals (RMMCH) with regards to their completeness, accuracy and clinical relevance.

Method: A retrospective review of 191 cranial ultrasound requests and reports was performed at two academic centres. A data collection sheet was developed by the principal investigator and supervisor guided by literature with regards to the information required within the cranial ultrasound report. A scoring method was then developed with a maximum score of 3 given for the request adequacy and of 14 for report adequacy.

Results: Only $49.74 \%$ of the requests met the criteria for an adequate request. The mean report adequacy score was 7.03 with a SD of (2.02). Fifty (50\%) of the reports had a maximum score of 7 . Overall $50.26 \%$ of the requests scored average and below average scores. The most commonly reported variable was the presence or absence of hydrocephalus and the least reported was resistive index. Results show that reports and requests are inadequate.

Note: A selection of conference abstracts: RSSA/SASPI Paediatric Imaging Congress, 03-06 November 2016, Spier Estate, Stellenbosch, South Africa. Faculty collaborators: Professor Kassa Darge (Body Imaging, University of Pennsylvania, Philadelphia,USA), Professor Edward Lee (Thoracic Imaging, Harvard University, USA), Professor Beverley Newman (Cardiac Imaging, Stanford University, California, USA), Professor Kimberly Applegate (Image Gently and Body Imaging, Emory University, Atlanta, USA) and Professor Savvas Andronikou (Thoracic Imaging, University of Bristol, UK) supported by South African Paediatric Radiologists, co-ordinated by Dr Jaishree Naidoo, President of the African Society of Paediatric Imaging and Head of Division of Paediatric Radiology, Charlotte Maxeke Johannesburg Academic Hospital. 